\title{
The Selberg Trace Formula for Bordered Riemann Surfaces
}

\author{
J. Bolte ${ }^{1}$ and F. Steiner \\ II. Institut für Theoretische Physik, Universität Hamburg, Luruper Chaussee 149, D-22761 \\ Hamburg, Germany
}

Received February 21, 1991

\begin{abstract}
A Selberg trace formula is derived for the Laplace-Beltrami operator on bordered Riemann surfaces with Dirichlet or Neumann boundary conditions, respectively, using a construction via the compact double of the surface, for which the standard trace formula is valid. Applications of the trace formula to spectral functions of the Laplace-Beltrami operators are discussed and their functional determinants are explicitly expressed in terms of various Selberg zeta functions. For Selberg's zeta function relevant to the Dirichlet boundary value problem a representation as a Dirichlet series is given, for which we conjecture conditional convergence even within the critical strip for $\operatorname{Re} s>\frac{1}{2}$.
\end{abstract}

\section{Introduction}

In recent years the Selberg trace formula [1-3] has become notably popular among physicists. There are two fields in physics, where Riemann surfaces occur and the trace formula has been successfully applied: quantum chaology $[4,5]$ and string theory [6]. In the first field it was discovered [4] that Gutzwiller's periodicorbit theory for the semiclassical quantization of a classically chaotic system becomes exact for a particle sliding freely on a Riemann surface of genus $g \geqq 2$ (Hadamard-Gutzwiller model). The corresponding periodic-orbit formula is just Selberg's trace formula. This then has been intensively applied there [5].

The second striking application of the Selberg trace formula has been string theory. In Polyakov's path integral approach [6], where the string partition function is given as an integral over all world sheets of the string, there occurs the functional determinant of the Laplace-Beltrami operator on the world sheet as a result of the integration over the embedding functions into space-time. It is possible to evaluate this determinant using the Selberg trace formula and express it through Selberg's zeta function $[6,7]$. Also, the ghost determinant appearing in string theory may be expressed analogously. In fact, the determinants

${ }_{1}^{1}$ Supported by Doktorandenstipendium der Universität Hamburg 
of higher-rank Laplace-like operators allow such a treatment, too [8]. Even in the case of the superstring a "super-analogue" of Selberg's trace formula has been derived and applied [9]. Thus the use of Selberg techniques has become a whole business in physics.

In this paper we derive a trace formula for bordered Riemann surfaces, that is we study the trace of certain integral operators associated with the LaplaceBeltrami operator obeying Dirichlet or Neumann boundary conditions. Our interest in that subject mainly stems from string theory, but we hope that one will be able to use the formula also in quantum chaology, e.g. to study the quantum Sinai billiard.

In string theory, one way to define off-shell string scattering amplitudes is to use a functional integral over bordered world sheets [10], the bordering curves being the incoming and outgoing string states of the scattering process. Then naturally the functional determinant of the Laplace-Beltrami operator occurs and the question arises, how to deal with it. Blau et al. [11] used for the first time a version of Selberg's trace formula for bordered surfaces, but restricted their attention to surfaces of genus zero.

Our purpose in this paper is to derive a trace formula for bordered Riemann surfaces of arbitrary genus. We proceed by constructing a compact Riemann surface through doubling the original one and by using the well-known trace formula for the cocompact case. To deal with the boundary conditions on the bordered surface properly, we divide the eigenfunctions of the Laplace-Beltrami operator on the doubled surface into symmetry classes according to their reflection property introduced by the doubling procedure. Similar considerations have been performed in special cases in [11-13]. Such a trace formula in the general context has first been derived by Venkov [14]. In this article we give a self-consistent derivation of the trace formula using similar methods as in [14] and also discuss some applications of it.

Our paper is organized as follows: First we explain the construction of the doubled surfaces and derive the trace formula. Then we study the trace of the heat kernel and the MP-zeta function of the Dirichlet-(Neumann-)Laplace-Beltrami operator and give some of their properties. In addition to the usual Selberg zeta function (on the doubled surface) we introduce functions that effectively take care of the correct boundary conditions. We are then in a position to express the functional determinant of the Dirichlet-(Neumann-)Laplace-Beltrami operator by these functions or by the respective heat kernels. These are the formulae that have been used in [15] to study the on-shell limit of off-shell string scattering amplitudes. Finally, we rearrange the trace formula and introduce a new Selberg zeta function for the Dirichlet problem, which can be identified with a combination of the previously introduced functions. In addition we discuss several properties of this zeta function.

\section{Derivation of the Trace Formula}

Before we start to derive the trace formula, we briefly recall how bordered Riemann surfaces are most conveniently dealt with [16]. The idea is to lift the discussion to an appropriately chosen compact surface, since the theory of compact Riemann surfaces is well developed and comparatively easy to handle. 
Let $\tilde{\Sigma}$ be a compact Riemann surface of genus $g$ and $d_{1}, \ldots, d_{n}$ conformal, non-overlapping discs on $\tilde{\Sigma}$. Then $\Sigma:=\tilde{\Sigma} \backslash\left\{d_{1}, \ldots, d_{n}\right\}$ is a bordered Riemann surface of signature $(g, n) . c_{i}:=\partial d_{i}$ are the $n$ components of $\partial \Sigma$. Now one takes a copy $I \Sigma$ of $\Sigma$, a mirror image, and glues both surfaces together along $\partial \Sigma$ and $\partial(I \Sigma)$. Technically this is done in terms of local coordinates in the following way: Let $z$ be a local coordinate in the neighbourhood of $P \in \Sigma$. Then $-\bar{z}$ is taken as the local coordinate in the neighbourhood of the mirror image $P^{\prime}$ of $P$ on $I \Sigma$. The corresponding points on $\partial \Sigma$ and $\partial(I \Sigma)$, respectively, are identified and thus have purely imaginary coordinates. The reflection $I: P \rightarrow P^{\prime}$ in $\partial \Sigma$ then is an anticonformal involution $\left(I^{2}=1\right)$ on the doubled surface $\hat{\Sigma}:=\Sigma \cup I \Sigma$. Furthermore $\Sigma=\hat{\Sigma} / I$, and $\hat{\Sigma}$ is a compact Riemann surface of genus $\hat{g}=2 g+n-1$. The uniformization theorem for compact Riemann surfaces now states that $\hat{\Sigma}$, for $\hat{g} \geqq 2$, may be represented as $\hat{\Sigma} \simeq \hat{\Gamma} \backslash \mathscr{H}$, where $\hat{\Gamma}$ is the Fuchsian group of $\hat{\Sigma}$ and $\mathscr{H}$ is the Poincaré upper half-plane, $\mathscr{H}=\{z=x+i y \mid y>0\}$, endowed with the hyperbolic metric $d s^{2}=y^{-2}\left(d x^{2}+d y^{2}\right) . \hat{\Gamma}$ then is a discrete subgroup of $\operatorname{PSL}(2, \mathbb{R})$ that solely consists of hyperbolic elements. In several circumstances it is advantageous to represent $\hat{\Sigma}$ by a fundamental domain $\hat{\mathscr{F}} \subset \mathscr{H}$ for $\hat{\Gamma}$.

To construct a convenient fundamental domain and representation of the involution $I$ on it, it is advantageous to view $\hat{\Sigma}$ as a symmetric Riemann surface with reflection symmetry $I$. For such surfaces the Fuchsian groups are well investigated by Sibner [17]. He shows that $\hat{\mathscr{F}}$ may be chosen as the interior of a fundamental polygon in $\mathscr{H}$ with $4 \hat{g}+2 n-2$ edges, which is symmetric with respect to the imaginary axes. The involution $I$ is being represented by $z \rightarrow-\bar{z}$, that is a reflection in the symmetry axis of $\hat{\mathscr{F}}$. One of the bordering curves, say $c_{n}$, is mapped onto the imaginary axis and the others are among the edges of the fundamental polygon. The advantage of this construction is that one can work directly on $\widehat{\mathscr{F}}$, with $I$, viewed as a mapping of complex numbers, being formally identical on $\hat{\Sigma}$ and $\hat{\mathscr{F}}$.

On $\mathscr{H}$ the Laplace-Beltrami operator takes the form $\Delta=y^{2}\left(\partial_{x}^{2}+\partial_{y}^{2}\right)$, hence it commutes with $I$. Therefore the eigenfunctions of $-\Delta$ can be simultaneously chosen as eigenfunctions of $I$. The odd functions (with respect to $I$ ) on $\hat{\Sigma}$ are exactly the (antisymmetric) continuations of the functions on $\Sigma$ that satisfy Dirichlet boundary conditions on $\partial \Sigma$, and the even functions are in the same way related to the functions on $\Sigma$ that satisfy Neumann boundary conditions. In this paper we will explicitly deal with the Dirichlet case and mention only from time to time how the Neumann case looks like. In the trace formula the difference is just a few signs. Thus from now on we concentrate on odd functions on $\hat{\Sigma}$ or $\hat{\mathscr{F}}$, respectively. They may be constructed from functions defined on the whole of $\mathscr{H}$ via Poincaré series. Let $f_{0} \in C(\mathscr{H})$ be continuous, then

$$
f(z):=\sum_{\gamma \in \hat{\Gamma}}\left[f_{0}(\gamma z)-f_{0}(\gamma I(z))\right]=\sum_{\gamma \in \hat{\Gamma}}\left[f_{0}(\gamma z)-f_{0}(\gamma(-\bar{z}))\right]
$$

is such an odd, $\hat{\Gamma}$-automorphic function, i.e. $f(\gamma z)=f(z)$ for all $\gamma \in \hat{\Gamma}$, and $f(I z)=-f(z)$.

In the Selberg trace formula one considers traces of integral operators, whose spectra are related to the spectrum of $-\Delta$. Let $\Phi \in C_{c}^{\infty}(\mathbb{R})$ be a smooth function with compact support. Then for $z, z^{\prime} \in \mathscr{H}$,

$$
k\left(z, z^{\prime}\right):=\Phi\left(\frac{\left|z-z^{\prime}\right|^{2}}{y y^{\prime}}\right)
$$


is called a point-pair invariant. For $M \in P S L(2, \mathbb{R})$, which acts on $z \in \mathscr{H}$ as a fractional linear transformation, it follows that $k\left(M z, M z^{\prime}\right)=k\left(z, z^{\prime}\right)$ and also $k\left(I z, I z^{\prime}\right)=k\left(z, z^{\prime}\right)$. Let $\psi \in C^{\infty}(\mathscr{H})$ be an eigenfunction of $-\Delta,-\Delta \psi=\lambda \psi$, then it is simultaneously an eigenfunction of the integral operator $L$ (see e.g. [2]),

$$
(L \psi)(z):=\int_{\mathscr{H}} d \mu\left(z^{\prime}\right) k\left(z, z^{\prime}\right) \psi\left(z^{\prime}\right)=\Lambda(\lambda) \psi(z),
$$

where $d \mu(z):=\frac{d x d y}{y^{2}}$ is the Poincaré measure on $\mathscr{H}$, and $\Lambda$ depends only on $\Phi$. Now we form the integral kernel

$$
\hat{K}\left(z, z^{\prime}\right):=\frac{1}{2} \sum_{\gamma \in \hat{\Gamma}}\left[k\left(z, \gamma z^{\prime}\right)-k\left(z, \gamma\left(-\bar{z}^{\prime}\right)\right)\right],
$$

which defines an integral operator on $L_{2}(\hat{\mathscr{F}})$. If $f \in L_{2}(\hat{\mathscr{F}})$ is an odd eigenfunction of $-\Delta$ with eigenvalue $\lambda$, then we compute

$$
\begin{aligned}
(\hat{L} f)(z): & =\int_{\hat{\mathscr{F}}} d \mu\left(z^{\prime}\right) \hat{K}\left(z, z^{\prime}\right) f\left(z^{\prime}\right) \\
& =\frac{1}{2}(L f)(z)-\frac{1}{2}(L f)(-\bar{z}) \\
& =\Lambda(\lambda) f(z),
\end{aligned}
$$

hence $f$ is also an eigenfunction of $\hat{L}$, with eigenvalue $\Lambda(\lambda)$. $-\Delta$ on $\hat{\Sigma}$ has a discrete spectrum, $0<\lambda_{1} \leqq \lambda_{2} \leqq \ldots$, and $\hat{K}$ can be expanded in (odd) eigenfunctions $\varphi_{n}$ of $-\Delta$,

$$
\hat{K}\left(z, z^{\prime}\right)=\sum_{n=1}^{\infty} \Lambda\left(\lambda_{n}\right) \varphi_{n}(z) \varphi_{n}\left(z^{\prime}\right) .
$$

As usual, one defines $\lambda=p^{2}+\frac{1}{4}, \Lambda(\lambda)=h(p)$, and gets as the trace of $\hat{L}$,

$$
\begin{aligned}
\operatorname{Tr} \hat{L}=\sum_{n=1}^{\infty} \Lambda\left(\lambda_{n}\right) & =\sum_{n=1}^{\infty} h\left(p_{n}\right) \\
& =\int_{\hat{\mathscr{F}}} d \mu(z) \hat{K}(z, z) \\
& =\frac{1}{2} \sum_{\gamma \in \hat{\Gamma}} \int_{\hat{\mathscr{F}}} d \mu(z) k(z, \gamma z)-\frac{1}{2} \sum_{\rho \in \hat{\Gamma} I} \int_{\hat{\mathscr{F}}} d \mu(z) k(z, \rho z) .
\end{aligned}
$$

The first sum on the r.h.s. of (7) is $\frac{1}{2}$ times the r.h.s. of the usual Selberg trace formula for the compact surface $\hat{\Sigma}$, and the result of its evaluation is well known to be $[1,2]$

$$
\begin{aligned}
\sum_{\gamma \in \hat{\Gamma}} \int_{\hat{\mathscr{F}}} d \mu(z) k(z, \gamma z)= & (\hat{g}-1) \int_{-\infty}^{+\infty} d p h(p) p \tanh (\pi p) \\
& +\sum_{\{\gamma\}_{p}} \sum_{k=1}^{\infty} \frac{l(\gamma)}{2 \sinh \left(\frac{k}{2} l(\gamma)\right)} g(k l(\gamma)) .
\end{aligned}
$$

In order that all the sums and integrals converge absolutely, $h(p)$ has to be an even function, holomorphic in the strip $|\operatorname{Im} p| \leqq \frac{1}{2}+\varepsilon, \varepsilon>0$, and has to decrease faster 
than $|p|^{-2}$ at infinity. $g(x):=\int_{-\infty}^{+\infty} \frac{d p}{2 \pi} h(p) e^{i p x}$ is the Fourier-transform of $h(p)$, and the sum over $\{\gamma\}_{p}$ runs over all primitive conjugacy classes in $\hat{\Gamma} . l(\gamma)$ is the length of the closed geodesic on $\hat{\Sigma}$ that corresponds to the hyperbolic conjugacy class $\{\gamma\}_{p}$.

We now evaluate the second sum on the r.h.s. of (7). To this end we first study the set $\hat{\Gamma} I$ of reflections. We divide the primitive reflections in $\hat{\Gamma} I$ into two classes as discussed in [11]. Here an element $\rho \in \hat{\Gamma} I$ is called primitive, if it cannot be written as a power of another reflection. Since $\rho \in \hat{\Gamma} I$ implies that $\rho^{2} \in \hat{\Gamma}$, the reflections were classified in [11] according to the properties of their squares. Three cases are to be distinguished:

- $\rho=\rho_{e}, \rho_{e}^{2}=1$. These are the pure reflections in a geodesic.

- $\rho=\rho_{i}, \rho_{i}^{2} \in\left\{C_{i}\right\}_{\hat{\Gamma}}, i=1, \ldots, n$. The $\left\{C_{i}\right\}_{\hat{\Gamma}}$ are the conjugacy classes of the $C_{i}$ in $\hat{\Gamma}$, which correspond to the closed geodesics $c_{i}$ on $\hat{\Sigma}$.

- $\rho=\rho_{p}, \rho_{p}^{2}$ being a primitive element in $\hat{\Gamma}$ and $\rho_{p}^{2} \notin\left\{C_{i}\right\} \hat{\Gamma}$.

There are, however, no pure reflections in $\hat{\Gamma}$ that do not lie in the second class. To see this write $\rho_{i}=\gamma_{i} I, \gamma_{i} \in \hat{\Gamma}$. As $\rho_{i} c_{i}=c_{i}$ it follows that $I c_{i}=\gamma_{i}^{-1} c_{i}$. Hence $\gamma_{i}$ is the hyperbolic transformation that identifies the edge of $\hat{\mathscr{F}}$ in one half of $\mathscr{H}$ with its corresponding edge, which, by the symmetric construction of $\hat{\mathscr{F}}$, lies in the other half of $\mathscr{H}$. Now, the $\gamma_{i}$ are the only transformations in $\hat{\Gamma}$ that identify edges in different halves of $\mathscr{H}$. Thus the $\rho_{i}$ are the only primitive pure reflections.

We now split the sum over the $\rho \in \hat{\Gamma} I$ according to the above classification and get all elements out of the primitive ones by summing over all powers of the primitive reflections. There one must only take odd powers, since an even power of a reflection is a hyperbolic transformation. We define $I_{\hat{\mathscr{F}}}(\rho):=\int_{\hat{\mathscr{F}}} d \mu(z) k(z, \rho z)$ as a shorthand and get

$$
\sum_{\rho \in \hat{\Gamma} I} I_{\hat{\mathscr{F}}}(\rho)=\sum_{i=1}^{n} \sum_{\rho_{i}} \sum_{k=0}^{\infty} I_{\hat{\mathscr{F}}}\left(\rho_{i}^{2 k+1}\right)+\sum_{\rho_{p}} \sum_{k=0}^{\infty} I_{\hat{\mathscr{F}}}\left(\rho_{p}^{2 k+1}\right) .
$$

With these expressions one can repeat the manipulations carried out in [11]:

$$
\begin{aligned}
\sum_{\rho} I_{\hat{\mathscr{F}}}\left(\rho^{2 k+1}\right) & =\sum_{\{\rho\}} \sum_{\sigma \in\{\rho\}} I_{\hat{\mathscr{F}}}\left(\sigma^{2 k+1}\right) \\
& =\sum_{\{\rho\}} \sum_{\gamma \in Z\left(\rho^{2}\right) \backslash \hat{\Gamma}} I_{\hat{\mathscr{F}}}\left(\gamma^{-1} \rho^{2 k+1} \gamma\right) \\
& =\sum_{\{\rho\}} \int_{Z\left(\rho^{2}\right) \backslash \mathscr{H}} d \mu(z) k\left(z, \rho^{2 k+1} z\right) .
\end{aligned}
$$

Here $Z\left(\rho^{2}\right):=\left\{\gamma \in \hat{\Gamma} \mid \gamma \rho^{2} \gamma^{-1}=\rho^{2}\right\}$ and $Z(\rho):=\left\{\gamma \in \hat{\Gamma} \mid \gamma \rho \gamma^{-1}=\rho\right\}$ are the centralizers of $\rho^{2}$ and $\rho$ respectively. One easily finds that they are equal, $Z(\rho)=Z\left(\rho^{2}\right)$.

We now conjugate $\rho^{2}$ in such a way that it acts as a dilatation by a factor $N\left(\rho^{2}\right)=e^{l\left(\rho^{2}\right)}$, where $l\left(\rho^{2}\right)$ is the length of the closed geodesic on $\hat{\Sigma}$ corresponding to $\left\{\rho^{2}\right\}$. Then $\rho$ itself acts as $\rho z=e^{l\left(\rho^{2}\right) / 2}(-\bar{z})$. A fundamental domain for the centralizer $Z\left(\rho^{2}\right)$ is given by $\left\{z \in \mathscr{H} \mid 1 \leqq y<N\left(\rho^{2}\right)\right\}$. Thus

$$
\begin{aligned}
\sum_{\rho \in \hat{\Gamma} I} \int_{\hat{\mathscr{F}}} d \mu(z) k(z, \rho z)= & 2 \sum_{\left\{\rho_{i}\right\}} \sum_{k=0}^{\infty} \int_{1}^{N\left(C_{t}\right)} \frac{d y}{y^{2}} \int_{0}^{\infty} d x \Phi\left(\frac{\left|z+N^{k}\left(C_{i}\right) \bar{z}\right|^{2}}{N^{k}\left(C_{i}\right) y^{2}}\right) \\
& +2 \sum_{\left\{\rho_{p}\right\}} \sum_{k=0}^{\infty} \int_{1}^{N\left(\rho_{p}^{2}\right)} \frac{d y}{y^{2}} \int_{0}^{\infty} d x \Phi\left(\frac{\left|z+N^{k+1 / 2}\left(\rho_{p}^{2}\right) \bar{z}\right|^{2}}{N^{k+1 / 2}\left(\rho_{p}^{2}\right) y^{2}}\right) .
\end{aligned}
$$


A manipulation well-known from the proof of the Selberg trace formula in the cocompact case leads to (see e.g. [2])

$$
\int_{1}^{N_{0}} \frac{d y}{y^{2}} \int_{0}^{\infty} d x \Phi\left(\frac{|z+N \bar{z}|^{2}}{N y^{2}}\right)=\frac{1}{2} \frac{\ln N_{0}}{N^{1 / 2}+N^{-1 / 2}} g(\ln N) .
$$

We use (12) in (11) and insert $N\left(\rho^{2}\right)=e^{l\left(\rho^{2}\right)}$. In the first sum of (11) we separate the $k=0$ term and introduce $L:=\sum_{i=1}^{n} l\left(c_{i}\right)$ as the total length of $\partial \Sigma$. The lengths $l\left(c_{i}\right)$ are twofold degenerate, since $C_{i}$ and $C_{i}^{-1}$ both have to be included into the sum. We collect all this and formulate the trace formula as a theorem.

Theorem. Let $h(p)$ be an even function, analytic in the strip $|\operatorname{Im} p| \leqq \frac{1}{2}+\varepsilon, \varepsilon>0$, and decreasing faster than $|p|^{-2}$ at infinity, with Fourier-transform $g(x)=\int_{-\infty}^{+\infty} \frac{d p}{2 \pi} h(p) e^{i p x}$. Let $\lambda_{n}=p_{n}^{2}+\frac{1}{4}$ be the eigenvalues with multiplicities $d_{n}$ of the Dirichlet-LaplaceBeltrami operator on the bordered Riemann surface $\Sigma$ of signature $(g, n)$, then $(\hat{g}=2 g+n-1, \hat{g} \geqq 2)$

$$
\begin{aligned}
\sum_{n=1}^{\infty} d_{n} h\left(p_{n}\right)= & \frac{\hat{g}-1}{2} \int_{-\infty}^{+\infty} d p h(p) p \tanh (\pi p)+\sum_{\{\gamma\}_{p}} \sum_{k=1}^{\infty} \frac{l(\gamma)}{4 \sinh (k l(\gamma) / 2)} g(k l(\gamma)) \\
& -\sum_{\left\{\rho_{p}\right\}} \sum_{k=0}^{\infty} \frac{l\left(\rho_{p}^{2}\right)}{4 \cosh \left[(k+1 / 2) l\left(\rho_{p}^{2}\right) / 2\right]} g\left((k+1 / 2) l\left(\rho_{p}^{2}\right)\right) \\
& -\frac{L}{4} g(0)-\sum_{i=1}^{n} \sum_{k=1}^{\infty} \frac{l\left(c_{i}\right)}{2 \cosh \left[k l\left(c_{i}\right) / 2\right]} g\left(k l\left(c_{i}\right)\right) .
\end{aligned}
$$

Remarks.

1. The function $h(p)$ has to satisfy the same conditions as in the cocompact case of (8), because the same manipulations have been done in the derivations of both formulae.

2. The case of Neumann boundary conditions can be treated in complete analogy to the Dirichlet case. One then has to study functions which are even under the reflection $I$ on $\hat{\Sigma}$. The relevant integral kernel for which one has to evaluate the trace is given by

$$
\hat{K}^{N}\left(z, z^{\prime}\right):=\frac{1}{2} \sum_{\gamma \in \hat{\Gamma}}\left[k\left(z, \gamma z^{\prime}\right)+k\left(z, \gamma\left(-\bar{z}^{\prime}\right)\right)\right] .
$$

The only difference to (13) is that the minus signs in front of the three last terms change into plus signs.

\section{Application to Heat Kernels, Zeta Functions and Determinants}

The first spectral function that we want to study with the help of the trace formula is the trace of the heat kernel for $\frac{\partial}{\partial t}-\Delta$. For $t>0$ this is defined by $(\mathrm{D}=$ Dirichlet $)$

$$
\theta_{\mathbf{D}}(t):=\operatorname{Tr} e^{t \Delta}=\sum_{n=1}^{\infty} d_{n} e^{-\lambda_{n} t}
$$


Inserting the function $h(p)=e^{-\left(p^{2}+\frac{1}{4}\right) t}$ into the trace formula (13), we obtain $\theta_{\mathrm{D}}(t)=\sum_{k=1}^{5} \theta_{\mathrm{D}}^{(k)}(t)$, where the terms on the r.h.s. are labelled according to their appearance in (13):

$$
\begin{aligned}
& \theta_{\mathrm{D}}^{(1)}(t)=\frac{\hat{g}-1}{4 \sqrt{\pi}} \frac{e^{-t / 4}}{t^{3 / 2}} \int_{0}^{\infty} d u \frac{u e^{-u^{2} / 4 t}}{\sinh (u / 2)}, \\
& \theta_{\mathrm{D}}^{(2)}(t)=\frac{1}{8 \sqrt{\pi}} \frac{e^{-t / 4}}{\sqrt{t}} \sum_{\{\gamma\}_{p}} \sum_{k=1}^{\infty} \frac{l(\gamma)}{\sinh (k l(\gamma) / 2)} e^{-k^{2} l^{2}(\gamma) / 4 t}, \\
& \theta_{\mathrm{D}}^{(3)}(t)=-\frac{1}{8 \sqrt{\pi}} \frac{e^{-t / 4}}{\sqrt{t}} \sum_{\left\{\rho_{p}\right\}} \sum_{k=0}^{\infty} \frac{l\left(\rho_{p}^{2}\right)}{\cosh \left((k+1 / 2) l\left(\rho_{p}^{2}\right) / 2\right)} e^{-k^{2} l^{2}\left(\rho_{p}^{2}\right) / 4 t}, \\
& \theta_{\mathrm{D}}^{(4)}(t)=-\frac{L}{8 \sqrt{\pi}} \frac{e^{-t / 4}}{\sqrt{t}}, \\
& \theta_{\mathrm{D}}^{(5)}(t)=-\frac{1}{4 \sqrt{\pi}} \frac{e^{-t / 4}}{\sqrt{t}} \sum_{i=1}^{n} \sum_{k=1}^{\infty} \frac{l\left(c_{i}\right)}{\cosh \left(k l\left(c_{i}\right) / 2\right)} e^{-k^{2} l^{2}\left(c_{i}\right) / 4 t} .
\end{aligned}
$$

The first thing to study is the small- $t$ asymptotics. One notices that the contributions $\theta_{\mathrm{D}}^{(2)}, \theta_{\mathrm{D}}^{(3)}$ and $\theta_{\mathrm{D}}^{(5)}$ decrease exponentially for $t \rightarrow 0+$. Thus only $\theta_{\mathrm{D}}^{(1)}$ and $\theta_{\mathrm{D}}^{(4)}$ are relevant for the small- $t$ asymptotics, giving

$$
\theta_{\mathrm{D}}(t)=\frac{\hat{g}-1}{2 t}-\frac{L}{8 \sqrt{\pi}} \frac{1}{\sqrt{t}}-\frac{1}{6}(\hat{g}-1)+O(\sqrt{t}), \quad t \rightarrow 0+.
$$

In the case of Neumann boundary conditions the term proportional to $t^{-\frac{1}{2}}$ changes its sign. The result (17) is exactly what one expects from the general expansion given by McKean and Singer [18].

The functional determinant of the Laplace-Beltrami operator will be defined by the method of zeta function regularization. Therefore we need to investigate the zeta function of Minakshisundaram-Pleijel (MP-zeta function), which is for $\operatorname{Re} s>1$ defined as

$$
\zeta_{\mathrm{D}}(s):=\sum_{n=1}^{\infty} d_{n} \lambda_{n}^{-s}
$$

In terms of this function the determinant of $-\Delta$ is defined to be $\operatorname{det}(-\Delta)_{\mathrm{D}}:=$ $e^{-\zeta_{\mathrm{D}}^{\prime}(0)}$. This definition requires an analytical continuation of $\zeta_{\mathrm{D}}$ to $s=0$, which is possible, because $\zeta_{\mathrm{D}}$ is a meromorphic function with only a simple pole at $s=1$.

We take $h(p)=\left(p^{2}+\left(\sigma-\frac{1}{2}\right)\right)^{-s}, \operatorname{Re} s, \operatorname{Re} \sigma>1$, to use it in the trace formula. Then $\zeta_{\mathrm{D}}(s)=\lim _{\sigma \rightarrow 1+} \sum_{n=1}^{\infty} d_{n} h\left(p_{n}\right)$. Again we label the terms on the r.h.s. of the trace formula by $\zeta_{\mathrm{D}}^{(1)}, \ldots, \zeta_{\mathrm{D}}^{(5)}$. They are given by

$$
\begin{aligned}
& \zeta_{\mathrm{D}}^{(1)}(s)=\frac{\hat{g}-1}{s-1} \frac{\pi}{2} \int_{0}^{\infty} d p \frac{\left(p^{2}+1 / 4\right)^{1-s}}{\cosh ^{2}(\pi p)}, \\
& \zeta_{\mathrm{D}}^{(2)}(s)=\frac{1}{2 \Gamma(s)} \lim _{\sigma \rightarrow 1+} I(s ; \sigma), \\
& \zeta_{\mathrm{D}}^{(3)}(s)=-\frac{1}{2 \Gamma(s)} \lim _{\sigma \rightarrow 1+} J(s ; \sigma),
\end{aligned}
$$




$$
\begin{aligned}
& \zeta_{\mathrm{D}}^{(4)}(s)=-\frac{L}{\sqrt{\pi}} 2^{2 s-4} \frac{\Gamma\left(s-\frac{1}{2}\right)}{\Gamma(s)}, \\
& \zeta_{\mathrm{D}}^{(5)}(s)=-\frac{1}{2 \Gamma(s)} \lim _{\sigma \rightarrow 1+} H(s ; \sigma),
\end{aligned}
$$

where we have introduced the functions $I(s ; \sigma)$ and $J(s ; \sigma)$, which are both entire functions of $s$ for $\operatorname{Re} \sigma>1$, whereas $H(s ; \sigma)$ is entire in $s$ for $\operatorname{Re} \sigma>0$. These functions are defined by

$$
\begin{aligned}
I(s ; \sigma):= & \frac{(2 \sigma-1)^{\frac{1}{2}-s}}{\sqrt{4 \pi}} \sum_{\{\gamma\}_{p}} \sum_{k=1}^{\infty} \frac{l}{\sinh \left(\frac{k}{2} l\right)}(k l)^{s-\frac{1}{2}} K_{s-\frac{1}{2}}\left(k l\left(\sigma-\frac{1}{2}\right)\right), \\
J(s ; \sigma):= & \frac{(2 \sigma-1)^{\frac{1}{2}-s}}{\sqrt{4 \pi}} \sum_{\left\{\rho_{p}\right\}} \sum_{k=0}^{\infty} \frac{l}{\cosh \left(\left(k+\frac{1}{2}\right) \frac{l}{2}\right)}\left(\left(k+\frac{1}{2}\right) l\right)^{s-\frac{1}{2}} \\
& \times K_{s-\frac{1}{2}}\left(\left(k+\frac{1}{2}\right) l\left(\sigma-\frac{1}{2}\right)\right), \\
H(s ; \sigma):= & \frac{(2 \sigma-1)^{\frac{1}{2}-s}}{\sqrt{\pi}} \sum_{i=1}^{n} \sum_{k=1}^{\infty} \frac{l_{i}}{\cosh \left(\frac{k}{2} l_{i}\right)}\left(k l_{i}\right)^{s-\frac{1}{2}} K_{s-\frac{1}{2}}\left(k l_{i}\left(\sigma-\frac{1}{2}\right)\right) .
\end{aligned}
$$

$K_{v}(z)$ is a modified Bessel function, and we drop the argument of $l(\gamma)$ and $l\left(\rho_{p}^{2}\right)$ whenever it may not lead to confusion; $l_{i}:=l\left(c_{i}\right)$. Equation (19) serves as an analytical continuation to $s=0$, since the pole term has been explicitly extracted in $\zeta_{D}^{(1)}$.

In principle one could now study $\zeta_{D}^{\prime}(0)$, but to do this properly we first investigate $I(0 ; \sigma), J(0 ; \sigma)$ and $H(0 ; \sigma)$ for $\operatorname{Re} \sigma>1$ or $\operatorname{Re} \sigma>0$ respectively. After rearranging the $k$-summations in (20) we arrive at

$$
\begin{aligned}
& I(0 ; \sigma)=-\ln \prod_{\{\gamma\}_{p}} \prod_{n=0}^{\infty}\left(1-e^{-(\sigma+n) l}\right)=-\ln Z(\sigma) \\
& J(0 ; \sigma)=-\ln \prod_{\left\{\rho_{p}\right\}} \prod_{n=0}^{\infty}\left(\frac{\left(1-e^{-l\left(n+\frac{1}{2} \sigma\right)}\right)\left(1+e^{-l\left(n+\frac{1}{2}(\sigma+1)\right)}\right)}{\left(1-e^{-l\left(n+\frac{1}{2}(\sigma+1)\right)}\right)\left(1+e^{-l\left(n+\frac{1}{2} \sigma\right)}\right)}\right)=:-\ln Y(\sigma) \\
& H(0 ; \sigma)=-\ln \prod_{i=1}^{n} \prod_{m=0}^{\infty}\left(\frac{1-e^{-l_{\imath}(\sigma+2 m)}}{1-e^{-l_{i}(\sigma+(2 m+1))}}\right)^{2}=:-\ln X(\sigma)
\end{aligned}
$$

$Z(\sigma)$ is the usual Selberg zeta function (on the doubled surface $\hat{\Sigma}$ ) and $Y(\sigma), X(\sigma)$ are two new functions similar to $Z(\sigma)$, that come from the additional terms in the trace formula, and which take care of the boundary conditions.

In a next step we would like to discuss the analytic properties of the new zeta functions $X(s)$ and $Y(s)$. Therefore we study the trace of the regularized resolvent of $-\Delta$, which can be obtained by the choice $h(p)=\left[p^{2}+\left(s-\frac{1}{2}\right)^{2}\right]^{-1}-$ $\left[p^{2}+\left(\sigma-\frac{1}{2}\right)^{2}\right]^{-1}$, for $\operatorname{Re} s, \operatorname{Re} \sigma>1$, in the trace formula. The 1.h.s. then gives

$$
\lim _{\sigma \rightarrow 1+} \sum_{n=1}^{\infty} d_{n} h\left(p_{n}\right)=\sum_{n=1}^{\infty} d_{n}\left\{\frac{1}{\lambda_{n}+s(s-1)}-\frac{1}{\lambda_{n}}\right\} \text {. }
$$


Before performing the limit $\sigma \rightarrow 1+$ one obtains from the trace formula, by expressing the r.h.s. in terms of $Z(s), Y(s)$ and $X(s)$,

$$
\begin{aligned}
\sum_{n=1}^{\infty} d_{n} & {\left[\frac{1}{\lambda_{n}+s(s-1)}-\frac{1}{\lambda_{n}+\sigma(\sigma-1)}\right] } \\
= & -(\hat{g}-1)[\psi(s)-\psi(\sigma)]+\frac{1}{2} \frac{1}{2 s-1}\left\{\frac{Z^{\prime}(s)}{Z(s)}-\frac{Y^{\prime}(s)}{Y(s)}-\frac{X^{\prime}(s)}{X(s)}\right\}-\frac{L}{4} \frac{1}{2 s-1} \\
& -\frac{1}{2} \frac{1}{2 \sigma-1}\left\{\frac{Z^{\prime}(\sigma)}{Z(\sigma)}-\frac{Y^{\prime}(\sigma)}{Y(\sigma)}-\frac{X^{\prime}(\sigma)}{X(\sigma)}\right\}+\frac{L}{4} \frac{1}{2 \sigma-1}
\end{aligned}
$$

where $\psi(z):=\Gamma^{\prime}(z) / \Gamma(z)$ denotes the digamma function. It is known how $Z(\sigma)$ behaves in the limit $\sigma \rightarrow 1+[7]$,

$$
\lim _{\sigma \rightarrow 1+}\left[\frac{1}{2 \sigma-1} \frac{Z^{\prime}(\sigma)}{Z(\sigma)}-\frac{1}{\sigma(\sigma-1)}\right]=\frac{1}{2} \frac{Z^{\prime \prime}(1)}{Z^{\prime}(1)}-1=: B,
$$

and thus the behaviour of $X(\sigma)$ and $Y(\sigma)$ may be deduced from the fact that the limit $\sigma \rightarrow 1+$ yields a finite result on the 1.h.s. of (23). From (21) it is clear that

$$
\frac{X^{\prime}(1)}{X(1)}=-\left.\frac{d}{d \sigma} H(0 ; \sigma)\right|_{\sigma=1}=\sum_{i=1}^{n} \sum_{k=1}^{\infty} 2 l_{i} \frac{e^{-k l_{i}}}{1+e^{-k l_{i}}}
$$

is finite and positive, as (21) converges for $\operatorname{Re} \sigma>0$ and all the summands are positive. Hence also the limit

$$
\lim _{\sigma \rightarrow 1+}\left\{\frac{1}{2 \sigma-1}\left[\frac{Z^{\prime}(\sigma)}{Z(\sigma)}-\frac{Y^{\prime}(\sigma)}{Y(\sigma)}\right]\right\}=: B+A
$$

exists, which together with (24) defines the constant $A$. From (26) one may draw the behaviour of the logarithmic derivative of $Y(\sigma)$ at $\sigma=1$,

$$
\frac{Y^{\prime}(\sigma)}{Y(\sigma)}=\frac{1}{\sigma-1}-A+1+O(\sigma-1), \quad \sigma \rightarrow 1 .
$$

Therefore $Y(\sigma)$ itself has a simple zero at $\sigma=1$ and $Y^{\prime}(1)$ is finite. We now define $\gamma_{D}:=(\hat{g}-1) \gamma+\frac{B+A}{2}-\frac{L}{4}-\frac{1}{2} \frac{X^{\prime}(1)}{X(1)}$, where $\gamma$ is Euler's constant. It can be shown that $\gamma_{D}=\operatorname{FP} \zeta_{D}(1):=\lim _{s \rightarrow 1}\left(\zeta_{D}(s)-\frac{1}{2} \frac{\hat{g}-1}{s-1}\right)$ is the finite part of $\zeta_{D}(s)$ at $s=1$. With all these definitions the limit $\sigma \rightarrow 1+$ of (23) yields

$$
\begin{aligned}
\sum_{n=1}^{\infty} d_{n}\left[\frac{1}{\lambda_{n}+s(s-1)}-\frac{1}{\lambda_{n}}\right]= & -(\hat{g}-1) \psi(s)-\frac{L}{4} \frac{1}{2 s-1}-\gamma_{D} \\
& +\frac{1}{2} \frac{1}{2 s-1}\left\{\frac{Z^{\prime}(s)}{Z(s)}-\frac{Y^{\prime}(s)}{Y(s)}-\frac{X^{\prime}(s)}{X(s)}\right\} .
\end{aligned}
$$

This regularized trace of the resolvent is a meromorphic function of $s$ and thus defines a meromorphic continuation of the function $X(s) Y(s)$ to all $s \in \mathbb{C}$. Knowing the analytic properties of the Selberg zeta function [2] one can obtain the poles and 
zeroes of $X(s) Y(s)$ from (28). Denote the eigenvalues of $-\Delta$ on $\hat{\Sigma}$ by $\tau_{n}=\frac{1}{4}+r_{n}^{2}$ with multiplicities $\delta_{n}$, then the non-trivial zeroes of $Z(s)$ are $s_{n}=\frac{1}{2} \pm i r_{n}$. Furthermore denote the Dirichlet eigenvalues as before by $\lambda_{n}=\frac{1}{4}+p_{n}^{2}$ with multiplicities $d_{n}$ and the Neumann eigenvalues by $\lambda_{n}^{(N)}=\frac{1}{4}+p_{n}^{(N) 2}$ with multiplicities $d_{n}^{(N)}$. Then $X(s) Y(s)$ has poles of order $d_{n}$ at $s_{n}=\frac{1}{2} \pm i p_{n}$ and zeroes at $s_{n}=0,1, \frac{1}{2} \pm i p_{n}^{(N)}$ of order $1,1, d_{n}^{(N)}$ respectively.

Another way of obtaining the analytic properties of $X(s) Y(s)$ is to express this product through determinant functions. Therefore define

$$
\mathscr{D}_{i}(z):=\operatorname{det}^{\prime}(-\Delta+z)_{i},
$$

where the index $i=D, N, \Delta$ indicates, whether we take the case of Dirichlet or Neumann boundary conditions on $\partial \Sigma$ or $-\Delta$ on the whole of $\hat{\Sigma}$, and the prime denotes the omission of possible zero modes. The obvious relation $\mathscr{D}_{\Delta}(z)=\mathscr{D}_{D}(z) \mathscr{D}_{N}(z)$ is fulfilled for all $z$. This can be obtained using the regularization

$$
\mathscr{D}_{D}(z)=\mathscr{D}_{D}(0) e^{\gamma_{D} z} \prod_{n=1}^{\infty}\left[\left(1+\frac{z}{\lambda_{n}}\right) e^{-\frac{z}{\lambda_{n}}}\right]^{d_{n}},
$$

which is valid for all $z$. The other determinant functions are defined analogously. Using the representation [7]

$$
Z(s)=s(s-1) \mathscr{D}_{\Delta}(s(s-1)) e^{2 C(\hat{g}-1)}\left[(2 \pi)^{1-s} e^{s(s-1)} G(s) G(s+1)\right]^{2(\hat{g}-1)},
$$

with $C:=\frac{1}{4}-\frac{1}{2} \ln 2 \pi-2 \zeta^{\prime}(-1), \zeta(s)$ denoting Riemann's zeta function, and $G(s)$ being Barnes' double gamma function [19], one derives by integrating (28) in $s$,

$$
X(s) Y(s)=X(1) Y^{\prime}(1) s(s-1) e^{-\frac{L}{2}(s-1)} \frac{\mathscr{D}_{D}(0)}{\mathscr{D}_{N}(0)} \frac{\mathscr{D}_{N}(s(s-1))}{\mathscr{D}_{D}(s(s-1))} .
$$

This can be pushed even a bit further by eliminating the unknown constants $X(1)$ and $Y^{\prime}(1)$. To do this we study the limit $s \rightarrow \infty$ in (32).

Since $X(s)$ and $Y(s)$ are defined by the Euler products (21), they both converge to unity for $s \rightarrow \infty$, i.e. $\lim _{s \rightarrow \infty} X(s) Y(s)=1$. On the r.h.s. of (32) there occur the determinant functions, for which the same limit has to be investigated. Sarnak showed [20], that if the trace of a given heat kernel has the small- $t$ asymptotics $\theta(t)=\frac{a}{t}+\frac{b}{\sqrt{t}}+c+O(\sqrt{t}), t \rightarrow 0+$, then the corresponding determinant function has the asymptotics

$$
\ln \operatorname{det}(-\Delta+z)=a z-(a z-c) \ln z+2 b \sqrt{\pi} \sqrt{z}+f(z), \quad z \rightarrow \infty,
$$

where $\lim _{z \rightarrow \infty} f(z)=0$. In our case this implies,

$$
\begin{aligned}
\ln \frac{\operatorname{det}(-\Delta+s(s-1))_{N}}{\operatorname{det}(-\Delta+s(s-1))_{D}} & =\ln \frac{s(s-1) \mathscr{D}_{N}(s(s-1))}{\mathscr{D}_{D}(s(s-1))} \\
& =\frac{L}{2} \sqrt{s(s-1)}+f(s) .
\end{aligned}
$$

Thus

$$
1=\lim _{s \rightarrow \infty} X(s) Y(s)=X(1) Y^{\prime}(1) \frac{\mathscr{D}_{D}(0)}{\mathscr{D}_{N}(0)} e^{\frac{L}{4}}
$$


We insert (35) into (32) and obtain the desired representation for $X(s) Y(s)$ in terms of determinant functions,

$$
X(s) Y(s)=s(s-1) \frac{\mathscr{D}_{N}(s(s-1))}{\mathscr{D}_{D}(s(s-1))} e^{-\frac{L}{4}(2 s-1)},
$$

which clearly exhibits the analytic properties that we already discussed above.

To evaluate the functional determinant of the Dirichlet-Laplace-Beltrami operator we require an analytic continuation of $X(s), Y(s)$ and $Z(s)$ to $s=1$. For Selberg's zeta function there is one available (see Eq. (40) below) ${ }^{2}$ and we want to recall this formula here. In exactly the same manner a similar representation for $X(s)$ and $Y(s)$ may be obtained as well. For $Z(s)$ one starts with McKean's integral representation [21],

$$
\frac{Z^{\prime}(s)}{Z(s)}=2(2 s-1) \int_{0}^{\infty} d t e^{-s(s-1) t} \theta_{D}^{(2)}(t), \quad \operatorname{Re} s>1 .
$$

The additional factor of two on the r.h.s. stems from the fact that our $\theta_{D}^{(2)}(t)$ is $\frac{1}{2}$ times McKean's $\vartheta(t)$.

We integrate this logarithmic derivative from $s>1$ to $\sigma>s$ and after that perform $\sigma \rightarrow \infty$. (Notice that $\lim _{\sigma \rightarrow \infty} Z(\sigma)=1$.) This gives for $\operatorname{Re} s>1$,

$$
Z(s)=\exp \left\{-2 \int_{0}^{\infty} \frac{d t}{t} \theta_{D}^{(2)}(t) e^{-s(s-1) t}\right\} .
$$

Splitting the region of integration and using ([22], p. 342)

$$
\int_{1}^{\infty} \frac{d t}{t} e^{-x t}=E_{1}(x)=-\gamma-\ln x-\sum_{n=1}^{\infty} \frac{(-x)^{n}}{n n !}
$$

in (38) yields

$$
\begin{aligned}
Z(s)= & s(s-1) \exp \left\{\gamma+\sum_{n=1}^{\infty} \frac{[s(1-s)]^{n}}{n n !}-2 \int_{0}^{1} \frac{d t}{t} \theta_{D}^{(2)}(t) e^{-s(s-1) t}\right. \\
& \left.-2 \int_{1}^{\infty} \frac{d t}{t} e^{-s(s-1) t}\left[\theta_{D}^{(2)}(t)-\frac{1}{2}\right]\right\} .
\end{aligned}
$$

The large- $t$ asymptotics that may be derived from the trace formula expressions, $\theta_{D}^{(2)}(t)=\frac{1}{2}+O\left(e^{-\alpha t}\right), t \rightarrow \infty, \alpha:=\min \left(\tau_{1}, \frac{1}{4}\right)$, shows that the integrals in the exponential of $(40)$ converge for $\operatorname{Re} s>\frac{1}{2}+\sqrt{\frac{1}{4}+(\operatorname{Im} s)^{2}-\alpha}$. Since $\alpha>0$, the representation (40) is valid in a neighbourhood of $s=1$.

In complete analogy to this reasoning one can also find a representation for $Y(s)$ that converges in the vicinity of $s=1$ and reads

$$
\begin{aligned}
Y(s)= & s(s-1) \exp \left\{\gamma+\sum_{n=1}^{\infty} \frac{[s(1-s)]^{n}}{n n !}+2 \int_{0}^{1} \frac{d t}{t} \theta_{D}^{(3)}(t) e^{-s(s-1) t}\right. \\
& \left.+2 \int_{1}^{\infty} \frac{d t}{t} e^{-s(s-1) t}\left[\theta_{D}^{(3)}(t)+\frac{1}{2}\right]\right\} .
\end{aligned}
$$

\footnotetext{
$\overline{2 \text { Formula (40) }}$ is an unpublished result of Aurich and Steiner
} 
(41) has the same region of convergence as (40). The representation (21) for $X(s)$ is valid for $s=1$ anyway, thus it can most easily be rewritten in terms of $\theta_{D}^{(5)}$. For $\operatorname{Re} s>\frac{1}{2}$ one gets

$$
X(s)=\exp \left\{2 \int_{0}^{\infty} \frac{d t}{t} e^{-s(s-1) t} \theta_{D}^{(5)}(t)\right\} .
$$

Equations (40), (41) and (42) together can now be used to express the determinant of $-\Delta$ in terms of $X(1), Y^{\prime}(1)$ and $Z^{\prime}(1)$ or through $\theta_{D}$ respectively.

To evaluate $\operatorname{det}^{\prime}(-\Delta)_{D}$ we have to differentiate (19) at $s=0$. Using the analytic properties of $I(s ; \sigma), J(s ; \sigma)$ and $H(s ; \sigma)$ derived above, we get

$$
\zeta_{D}^{\prime}(0)=(\hat{g}-1) C+\frac{L}{8}+\ln \sqrt{X(1)}+\frac{1}{2} \lim _{\sigma \rightarrow 1+}[I(0 ; \sigma)-J(0 ; \sigma)],
$$

where we used the well-known result $[6,7] \zeta_{D}^{(1) \prime}(0)=(\hat{g}-1) C$. As $Y(\sigma)$ and $Z(\sigma)$ both have simple zeroes at $\sigma=1$, the result for the determinant is

$$
\operatorname{det}^{\prime}(-\Delta)_{D}=\sqrt{\frac{Z^{\prime}(1)}{X(1) Y^{\prime}(1)}} \exp \left[-(\hat{g}-1) C-\frac{L}{8}\right] .
$$

Using (40), (41) and (42) the prefactor of the exponential can be expressed through the trace of the heat kernel,

$$
\operatorname{det}^{\prime}(-\Delta)_{D}=\exp \left\{-\int_{0}^{\infty} \frac{d t}{t}\left[\theta_{D}^{(2)}(t)+\theta_{D}^{(3)}(t)+\theta_{D}^{(5)}(t)\right]\right\} \exp \left[-(\hat{g}-1) C-\frac{L}{8}\right]
$$

In [15] we have used (45) to express a part of the integrand in the formula for the string scattering amplitudes in terms of the lengths $l_{i}$ of the bordering curves $c_{i}$, that are the external string states of the scattering process.

The case of Neumann boundary conditions can easily be derived from (35),

$$
\begin{aligned}
\operatorname{det}^{\prime}(-\Delta)_{N} & =\operatorname{det}^{\prime}(-\Delta)_{D} X(1) Y^{\prime}(1) e^{\frac{L}{4}} \\
& =\sqrt{X(1) Y^{\prime}(1) Z^{\prime}(1)} \exp \left[-(\hat{g}-1) C+\frac{L}{8}\right] .
\end{aligned}
$$

\section{Selberg's Zeta Function for the Dirichlet Problem}

One can rearrange the trace formula (13) to yield a form that is more reminiscent of a periodic-orbit formula in the sense of [4]. (For an explicit example, see e.g. [23].) The second sum on the r.h.s. of (13) runs over the conjugacy classes $\left\{\rho_{p}\right\}$, where $\rho_{p}^{2} \in \hat{\Gamma}$ is primitive. Therefore the corresponding conjugacy class also occurs in the first sum over all primitive conjugacy classes in $\hat{\Gamma}$. It is thus natural to combine the contributions of each such conjugacy class from both sums into a single sum. As these classes correspond to closed geodesics on $\hat{\Sigma}$ that are symmetric w.r.t. the reflection $I$ we call them $b_{s}$. One also notes that $l\left(\rho_{p}^{2}\right)$ is twice the length of the geodesic on $\Sigma$; therefore we introduce $l\left(\rho_{p}^{2}\right)=: 2 l\left(b_{s}\right)$.

The remainder of the first sum on the r.h.s. of (13) consists of a summation over the closed geodesics that are not symmetric under $I$ - we call these geodesics $b_{n}-$ and over the bordering curves $c_{i}$. On $\Sigma$ the $b_{n}$ 's and their mirror images under 
I yield the same geodesic. We account for that fact by introducing a factor of two in the summation and counting each geodesic only once.

One notices that the inner $k$-summation in the two contributions to the sum over the $b_{s}$ 's are over even and odd integers respectively and thus can be combined into a single summation. Completing all this we arrive at

$$
\begin{aligned}
\sum_{n=1}^{\infty} d_{n} h\left(p_{n}\right)= & \frac{(\hat{g}-1)}{2} \int_{-\infty}^{+\infty} d p h(p) p \tanh (\pi p)-\frac{L}{4} g(0) \\
& +\sum_{b_{n}} \sum_{k=1}^{\infty} \frac{l\left(b_{n}\right) g\left(k l\left(b_{n}\right)\right)}{e^{k l\left(b_{n}\right) / 2}-e^{-k l\left(b_{n}\right) / 2}}+\sum_{b_{s}} \sum_{k=1}^{\infty} \frac{(-1)^{k} l\left(b_{s}\right) g\left(k l\left(b_{s}\right)\right)}{e^{k l\left(b_{s}\right) / 2}-(-1)^{k} e^{-k l\left(b_{s}\right) / 2}} \\
& +\sum_{i=1}^{n} \sum_{k=1}^{\infty} \frac{2 l_{i} e^{-k l_{i} / 2}}{e^{k l_{l}}-e^{-k l_{i}}} g\left(k l_{i}\right) .
\end{aligned}
$$

Introducing $\chi_{b}=1$ for $b=b_{n}$ and $\chi_{b}=-1$ for $b=b_{s}$ and suppressing the argument of $l(b)$ yields the alternative form of the trace formula

$$
\begin{aligned}
\sum_{n=1}^{\infty} d_{n} h\left(p_{n}\right)= & \frac{(\hat{g}-1)}{2} \int_{-\infty}^{+\infty} d p h(p) p \tanh (\pi p)-\frac{L}{4} g(0) \\
& +\sum_{b \neq c_{i}} \sum_{k=1}^{\infty} \frac{\left.\chi_{b}^{k} l g(k l)\right)}{e^{k l / 2}-\chi_{b}^{k} e^{-k l / 2}}+\sum_{i=1}^{n} \sum_{k=1}^{\infty} \frac{2 l_{i} e^{-k l_{\imath} / 2}}{e^{k l_{i}}-e^{-k l_{l}}} g\left(k l_{i}\right) .
\end{aligned}
$$

The zeta functions $X(s), Y(s)$ and $Z(s)$ were obtained after inserting $h(p)=$ $\left[p^{2}+(s-1 / 2)^{2}\right]^{-1}-\left[p^{2}+(\sigma-1 / 2)^{2}\right]^{-1}, \operatorname{Re} s, \operatorname{Re} \sigma>1$, into the trace formula (13). Using the same function $h(p)$ in (48) yields

$$
\sum_{n=1}^{\infty} d_{n} h\left(p_{n}\right)=-(\hat{g}-1) \psi(s)-\frac{L}{4} \frac{1}{2 s-1}+\frac{1}{2 s-1}\left[\frac{\tilde{Z}_{D}^{\prime}(s)}{\tilde{Z}_{D}(s)}+\frac{Z_{B}^{\prime}(s)}{Z_{B}(s)}\right]-(s \rightarrow \sigma),
$$

where we have defined

$$
\begin{array}{ll}
\tilde{Z}_{D}(s):=\prod_{b \neq c} \prod_{n=0}^{\infty}\left(1-\chi_{b}^{n+1} e^{-l(s+n)}\right), & \operatorname{Re} s>1, \\
Z_{B}(s):=\prod_{i=1}^{n} \prod_{k=0}^{\infty}\left(1-e^{-l_{i}(s+2 k+1)}\right)^{2}, & \operatorname{Re} s>0 .
\end{array}
$$

The product $Z_{D}(s):=Z_{B}(s) \cdot \tilde{Z}_{D}(s)$ now is Selberg's zeta function for the Dirichlet problem on the bordered surface $\Sigma$. Comparing this definition and (49) with (23) shows that

$$
Z_{D}^{2}(s)=\frac{Z(s)}{X(s) Y(s)}
$$

In terms of $Z_{D}(s)$ we obtain for the trace of the regularized resolvent

$$
\sum_{n=1}^{\infty} d_{n}\left[\frac{1}{\lambda_{n}+s(s-1)}-\frac{1}{\lambda_{n}}\right]=-(\hat{g}-1) \psi(s)-\frac{L}{4} \frac{1}{2 s-1}-\gamma_{D}+\frac{1}{2 s-1} \frac{Z_{D}^{\prime}(s)}{Z_{D}(s)} .
$$

The analytic properties of $X(s), Y(s)$ and $Z(s)$ (see the discussion after (28)) show that $Z_{D}(s)$ is an entire function with (trivial) zeroes of order $(\hat{g}-1)(2 k+1)$ at 
$s=-k, k \in \mathbb{N}_{0}$, and (non-trivial) zeroes of order $d_{n}$ at $s=\frac{1}{2} \pm i p_{n}, \lambda_{n}=\frac{1}{4}+p_{n}^{2}$ being the Dirichlet-eigenvalues of $-\Delta$ on $\Sigma$ with multiplicities $d_{n}$. These properties are explicitly seen in the representation

$$
Z_{D}(s)=\mathscr{D}_{D}(s(s-1)) e^{\frac{L}{8}(2 s-1)}\left[(2 \pi)^{1-s} e^{C+s(s-1)} G(s) G(s+1)\right]^{\hat{g}-1} .
$$

The functional equation of $Z(s)$ (see e.g. [2]) together with (36) shows that $Z_{D}(s)$ satisfies the functional equation

$$
Z_{D}(s)=Z_{D}(1-s) \exp \left\{2 \pi(\hat{g}-1) \int_{0}^{s-\frac{1}{2}} d w w \tan (\pi w)+\frac{L}{4}(2 s-1)\right\} .
$$

Instead of the Euler products (50) we want to find a representation for $Z_{D}(s)$ in terms of a Dirichlet series which seems to be more practical to treat. The idea to do this goes back to McKean [21] and has in a similar context been exploited by Berry and Keating [24]. We use the relation

$$
\prod_{n=0}^{\infty}\left(1-y x^{n}\right)=1+\sum_{m=1}^{\infty}(-1)^{m} \frac{x^{\frac{1}{2} m(m-1)}}{\prod_{r=1}^{m}\left(1-x^{r}\right)} y^{m},
$$

which is due to Euler [25]. Both sides converge absolutely for $|x|<1$ and $y \in \mathbb{C}$. For $\tilde{Z}_{D}(s)$ we take $x=\chi_{b} e^{-l}, y=\chi_{b} e^{-s l}$ and for $Z_{B}(s) x=e^{-2 l_{i}}, y=e^{-l_{i}(s+1)}$, and perform the inner products in (50) using (55),

$$
Z_{D}(s)=\prod_{b}\left\{1+\sum_{k=1}^{\infty} a_{k}^{(b)} e^{-s k l(b)}\right\} .
$$

Here the product over $b$ extends over all closed geodesics, where the $c_{i}$ 's occur with multiplicities two. In (56) we introduced the coefficients

$$
\begin{aligned}
& \text { - } b \neq c_{i}: \quad a_{k}^{(b)}:=(-1)^{k} \frac{\chi_{b}^{\frac{k}{2}(k+1)} e^{-\frac{k}{2}(k-1) l(b)}}{\prod_{r=1}^{k}\left(1-\chi_{b}^{r} e^{-r l(b)}\right)}, \\
& \text { - } b=c_{i}: \quad a_{k}^{\left(c_{i}\right)}:=(-1)^{k} \frac{e^{-k^{2} l_{i}}}{\prod_{r=1}^{k}\left(1-e^{-2 r l_{l}}\right)} .
\end{aligned}
$$

To evaluate the product in (56) we introduce so-called "pseudo-orbits" $\alpha$ as formal sums of primitive closed geodesics $b$,

$$
\alpha:=m_{1} b_{1} \oplus \cdots \oplus m_{k} b_{k}, \quad m_{i} \in \mathbb{N}_{0} .
$$

The length $L_{\alpha}$ of this pseudo-orbit is defined to be

$$
L_{\alpha}:=m_{1} l\left(b_{1}\right)+\cdots+m_{k} l\left(b_{k}\right) .
$$

We also introduce the norm $N_{\alpha}:=e^{L_{\alpha}}$. These notions allow us to perform the product in (56) to give

$$
Z_{D}(s)=\sum_{\alpha} a_{\alpha} N_{\alpha}^{-s}, \quad \operatorname{Re} s>1,
$$

where the sum runs over all possible pseudo-orbits that may be formed out of the closed geodesics $b$ on $\Sigma$ according to (58), including the "null-orbit" with $a_{0}=1=N_{0}$. Furthermore, $a_{\alpha}:=\prod_{r=1}^{k} a_{m_{r}}^{\left(b_{r}\right)}$.

Equation (60) is a representation of $Z_{D}(s)$ as a Dirichlet series. Such series converge absolutely in right half-planes $\operatorname{Re} s>\sigma_{A}$ and conditionally for $\operatorname{Re} s>\sigma_{C}$, $\sigma_{A} \geqq \sigma_{C}$. One can derive a similar formula like (60) also for $Z(s)$, where 
$\sigma_{A}=1$. The location of the abscissa of absolute convergence in the latter case is a consequence of the zero of $Z(s)$ at $s=1$ due to the existence of the eigenvalue $\tau_{0}=0 . Z_{D}(s)$, however, has no zero at $s=1$ and, in addition, the $\chi_{b}$ 's, which are not present for $Z(s)$, introduce alternating signs into the series. Thus one may conjecture that the Dirichlet series (60) converges conditionally also for some $\operatorname{Re} s \leqq 1$. The absolute convergence of (60) is not changed by the manipulations done, therefore $\sigma_{A}=1$. If there are no small eigenvalues $0<\lambda_{n}<\frac{1}{4}$, there are no zeroes of $Z_{D}(s)$ with $\operatorname{Re} s>\frac{1}{2}$. Our conjecture therefore is that in this case $\sigma_{C}=\frac{1}{2}$. This would mean that one could evaluate (60) numerically, knowing the geodesic length spectrum of $\Sigma$, for $\operatorname{Re} s=\frac{1}{2}+\varepsilon, \varepsilon$ positive but arbitrarily small, and could identify the zeroes $s_{n}=\frac{1}{2} \pm i p_{n}$ as minima of $Z_{D}(s)$ in the vicinity of the critical line $\operatorname{Re} s=\frac{1}{2}$. Thus one could compute the eigenvalues for the Dirichlet-Laplace-Beltrami operator on $\Sigma$.

\section{Summary}

In this paper we derived a Selberg trace formula for bordered Riemann surfaces. This formula allowed us to express functions of the eigenvalues of the LaplaceBeltrami operator, endowed with either Dirichlet or Neumann conditions on the surface's boundary, through the lengths of the closed geodesics on the compact double of the surface. On the other hand this could be viewed as a trace formula for reflection symmetric Riemann surfaces concerning the spectral problem of the Laplace-Beltrami operator on either even or odd functions under the symmetry operation.

We discussed spectral functions of $-\Delta$ for both Dirichlet and Neumann boundary conditions and evaluated the respective functional determinants. In addition we gave an expression that involved the relation between determinant functions of either cases.

Since in our final formula (45) the dependence of the determinant on the lengths of the bordering curves is made explicit, this result could be used in string theory to study the dependence of scattering amplitudes on the lengths of the external string states [15].

Finally we have investigated the Selberg zeta function $Z_{D}(s)$ for the Dirichlet problem on the bordered surface $\Sigma$. Rearranging the trace formula in such a way that it looks like a periodic-orbit formula for $\Sigma$, we could represent $Z_{D}(s)$ by an Euler product of the usual type. Rewriting this as a Dirichlet series, we were led to the hypothesis that we have achieved conditional convergence for that series left of the abscissa of absolute convergence $\operatorname{Re} s=1$ up to just before the critical line $\operatorname{Re} s=\frac{1}{2}$. This fact may be used in the context of periodic-orbit quantization of classically chaotic systems to compute the eigenvalues of a Sinai-billiard-like system, i.e. the free motion of a particle on a surface with holes.

\section{References}

1. Selberg, A.: J. Indian Math. Soc. 20, 47-87 (1956)

2. Hejhal, D.A.: The Selberg Trace Formula for $\operatorname{PSL}(2, \mathbb{R})$, Vol. 1. Springer Lecture Notes in Mathematics 548 (1976)

3. Elstrodt, J.: Jahresberichte d. Dt. Math. Vereinigung 83, 45-77 (1981) 
4. Gutzwiller, M.C.: Phys. Rev. Lett. 45, 150-153 (1980); Contemp. Math. 53, 215-251 (1986)

5. Aurich, R., Sieber, M., Steiner, F.: Phys. Rev. Lett. 61, 483-487 (1988) ; Aurich, R., Steiner, F.: Physica D39, 169-193 (1989)

6. D'Hoker, E., Phong, D.H.: Nucl. Phys. B269, 205-234 (1986); Gilbert, G.: Nucl. Phys. B277, 102-124 (1986); Namazie, M.A., Rajeev, S.: Nucl. Phys. B277, 332-348 (1986); D’Hoker, E., Phong, D.H.: Rev. Mod. Phys. 60, 917-1065 (1988)

7. Steiner, F.: Phys. Lett. 188B, 447-454 (1987)

8. D'Hoker, E., Phong, D.H.: Commun. Math. Phys. 104, 537-545 (1986); Bolte, J., Steiner, F.: Commun. Math. Phys. 130, 581-597 (1990); Oshima, K.: Phys. Rev. D41, 702-703 (1990)

9. Grosche, C.: Commun. Math. Phys. 133, 433-485 (1990)

10. Cohen, A., Moore, G., Nelson, P., Polchinski, J.: Nucl. Phys. B267, 143-157 (1986); Cohen, A., Moore, G., Nelson, P., Polchinski, J.: Nucl. Phys. B281, 127-144 (1987)

11. Blau, S.K., Clements, M.: Nucl. Phys. B284, 118-130 (1987)

12. Venkov, A.B.: Math. USSR Izv. 12, 448-462 (1978)

13. Guillopé, L.: Duke Math. J. 53, 827-848 (1986)

14. Venkov, A.B.: Spectral Theory of Automorphic Functions. Proc. Steklov Math. Inst. 153 (1981)

15. Bolte, J., Steiner, F.: Nucl. Phys. B, 361, 451-468 (1991)

16. Fay, J.D.: Theta Functions on Riemann Surfaces. Springer Lecture Notes in Mathematics 352 (1973)

17. Sibner, R.: Am. J. Math. 90, 1237-1259 (1968)

18. McKean, H.P., Singer, I.M.: J. Diff. Geom. $143-69$ (1967)

19. Barnes, E.W.: Quart. J. Pure and Appl. Math. 31, 264-314 (1900)

20. Sarnak, P.: Commun. Math. Phys. 110, 113-120 (1987)

21. McKean, H.P.: Commun. Pure Appl. Math. 25, 225-246 (1972)

22. Magnus, W., Oberhettinger, F., Soni, R.P.: Formulas and Theorems for the Special Functions of Mathematical Physics. Berlin Heidelberg New York: Springer 1966

23. Sieber, M., Steiner, F.: Physica D44, 248-266 (1990)

24. Berry, M.V., Keating, J.P.: J. Phys. A23, $4839-4849$ (1990)

25. Euler, L.: Introductio in Analysin Infinitorum $\$ \$ 306-307$ (1748)

Note added in proof. After submission of this paper formula (40) has been published in [26]. In the meantime the analytic continuation of various Selberg zeta functions into the critical strip, $\operatorname{Re} s<1$, using the Dirichlet series representation, and applications in quantum chaology have been discussed in detail in [27]. The conjecture on the conditional convergence should be modified by stating that the optimal value $\sigma_{C}=\frac{1}{2}$ for the abscissa of conditional convergence is conjectured to hold only for Riemann surfaces which are generated by non-arithmetic Fuchsian groups. See [28] for more details.

26. Aurich, R., Steiner, F.: Phys. Rev. A46, 771-781 (1992)

27. Matthies, C., Steiner, F.: Phys. Rev. A44, R7877-R7880 (1991); Aurich, R., Steiner, F.: Proc. Roy. Soc. London A437, 693-714 (1992); Aurich, R., Bolte, J., Matthies, C., Sieber, M., Steiner, F.: Physica D63, 71-86 (1993)

28. Bolte, J.: Some Studies on Arithmetical Chaos in Classical and Quantum Mechanics, DESY report, DESY 93-044 (April 1993), Int. J. Mod. Phys. B (in print) 